\begin{tabular}{|c|l|}
\hline Title & Direct Electron-Diffraction Evidence of Charge Density-Wave Formation in NbSe3 \\
\hline Author(s) & Tsutsumi, K.; Takagaki, T.; Y amamoto, M.; Shiozaki, Y.; I do, M.; Sambongi, T.; Y amay a, K.; A be, Y. \\
\hline Citation & $\begin{array}{l}\text { Physical Review Letters, 39(26), 1675-1676 } \\
\text { https://doi.org/10.1103/PhysRevLett.39.1675 }\end{array}$ \\
\hline Issue Date & 1977-12-26 \\
\hline Doc URL & http://hdl.handle.net/2115/56811 \\
\hline Rights & O1977 A merican Physical Society \\
\hline Type & article \\
\hline File Information & PhysRevLett.39.1675.pdf \\
\hline
\end{tabular}

Instructions for use 
Lett. 30,220 (1973).

${ }^{21}$ R. Chang and L. M. Falicov, J. Phys. Chem. Solid 32, 465 (1971), showed that a pseudopotential procedure which yields satisfactory lattice-vibrational spectra predicts negative (unphysical) values for the heat of formation of vacancies in metals such as $\mathrm{Al}$.

\title{
Direct Electron-Diffraction Evidence of Charge-Density-Wave Formation in $\mathrm{NbSe}_{3}$
}

\author{
K. Tsutsumi, T. Takagaki, M. Yamamoto, Y. Shiozaki, M. Ido, and T. Sambongi \\ Department of Physics, Hokkaido University, Sapporo 060, Japan \\ and \\ K. Yamaya and Y. Abe \\ Department of Nuclear Engineering, Hokkaido University, Sapporo 060, Japan
}

(Received 12 August 1977)

\begin{abstract}
In $\mathrm{NbSe}_{3}$ one-dimensional diffuse scattering was observed above $140 \mathrm{~K}$ and the satellite spots at a lower temperature. They correspond to the Kohn anomaly and the superlattice coupled with charge-density wave, respectively. The superlattice was found to be incommensurate, with the period $a^{\prime}=a, b^{\prime} \simeq 4.1 b$, and $c^{\prime}=c$ 。
\end{abstract}

Recently, the transition-metal trichalcogenides $M X_{3}\left(M: \mathrm{Nb}, \mathrm{Ta}_{i} X: \mathrm{S}, \mathrm{Se}\right)$ have been the subjects of several investigations. ${ }^{1-6}$ These needle-shaped materials have the chain structure of the transition-metal atoms surrounded by the chalcogen atoms along the needle axis. The distance between metal atoms along the chain is much shorter than the interchain distances. Therefore the interactions between chains seem to be weak and one-dimensional behavior is expected in $M X_{3}$.

One of the $M X_{3}$ family, $\mathrm{NbSe}_{3}$, is characterized by two cusps in the temperature variation of the electrical resistivity along the $b$ axis (needle axis) at 59 and $143 \mathrm{~K}$. These anomalies have been explained by assuming the formation of a charge-density wave (CDW)..$^{1-3}$ Especially the suppression of the two peaks in the resistivity by strong electrical fields is explained as Zener breakdown across the very small gaps at the Fermi surface induced by the formation of CDW. ${ }^{3}$ In this Letter we present the first direct evidence of CDW formation, by electron diffraction above $130 \mathrm{~K}$.

$\mathrm{NbSe}_{3}$ was synthesized by direct reaction of $\mathrm{Nb}$ and Se without carriers. The morphology and the resistivity anomalies of our crystals were the same as those reported by Haen et al . ${ }^{2}$ The residual resistivity ratio was $\sim 70$. It was found from $x$-ray diffraction studies with both the precession camera and the counter method, that the $(0 k 0)$ reflections were missing when $k=2 n+1$. This extinction rule indicates that the space group of $\mathrm{NbSe}_{3}$ is either $P 2_{1}$ or $P 2_{1} / m$, which have higher symmetry than $P m$ given by Meerschaut and Rouxel. ${ }^{4}$ It seems that the presence of the $2_{1}$ screw axis along the needle axis is a common feature of $M X_{3}{ }^{7 * 9}$ The electron diffraction of $\mathrm{NbSe}_{3}$ was performed with the acceleration voltage of $100 \mathrm{kV}$. The sample holder was kept at $130 \mathrm{~K}$. But the temperature of the individual crystal may be slightly higher as a result of irradiation by the electron beam, though a heat link to the sample holder would release some of the heat.

Figure 1 shows the $b^{*}-c *$ plane of the reciprocal space above $140 \mathrm{~K}$. The anomaly in diffuse scattering can be clearly seen to be one-dimensional. The position of this one-dimensional anomaly along the $b^{*}$ axis is $(0.244 \pm 0.004) b *$, which is a little smaller than the commensurate value of $0.25 b *$. This incommensurate streak

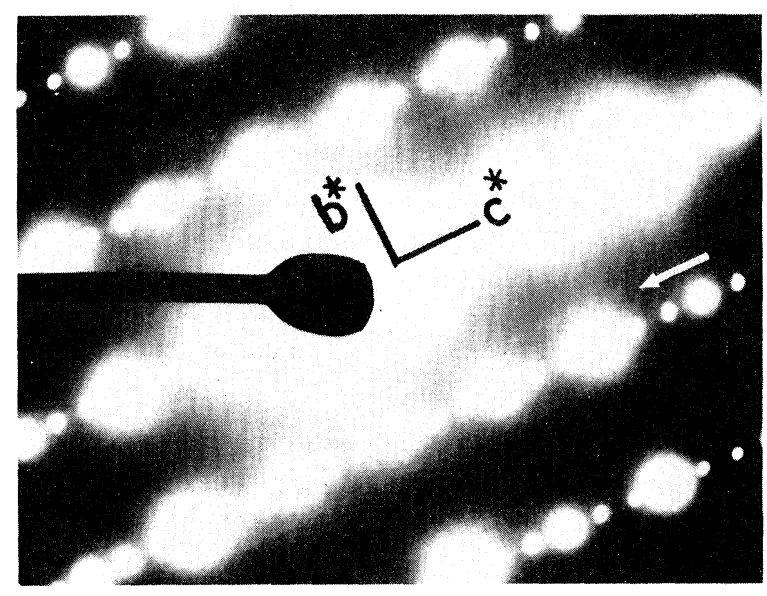

FIG. 1. Electron diffraction pattern of $\mathrm{NbSe}_{3}$ above $140 \mathrm{~K}$, showing one-dimensional anomaly in diffuse scattering (marked by arrows). 
corresponds to the Kohn anomaly. In Fig. 2, which show s the $a^{*}-b^{*}$ plane below $140 \mathrm{~K}$, the satellite spots are exactly at the position of the Kohn anomaly. From a similar observation on the $b^{*}-c^{*}$ plane below $140 \mathrm{~K}$, it was found that the satellite spots have no $a^{*}$ and $c^{*}$ components, at least at this temperature. These observations establish that an incommensurate superlattice with $a^{\prime}=a, b^{\prime} \simeq 4.1 b$, and $c^{\prime}=c$ occurs in $\mathrm{NbSe}_{3}$. The incommensurability itself is a strong support of the CDW mechanism. Thus our electron diffraction experiments confirmed the existence of $\mathrm{CDW}$ in $\mathrm{NbSe}_{3}$.

We reported that $\mathrm{TaS}_{3}$ undergoes the Peierls transition at $220 \mathrm{~K} .{ }^{6}$ Comparing the two materials, the following points are worth noting:

(i) The low-temperature phase of $\mathrm{TaS}_{3}$ has the commensurate superperiod of $c^{\prime}=4 c$ within the accuracy of $1 \%$, while it is incommensurate in $\mathrm{NbSe}_{3}$. (Note that the chain axis is the $b$ axis in $\mathrm{NbSe}_{3}$ and the $c$ axis in $\mathrm{TaS}_{3}$. )

(ii) From the nearly equal periods of the onedimensional modulation, it is expected that the Fermi surfaces of these materials have common features in the normal state, e.g., nearly the same relative position in the Brillouin zone.

(iii) $\mathrm{TaS}_{3}$ is semiconducting below the transition temperature, while $\mathrm{NbSe}_{3}$ remains metallic. The Fermi surface of $\mathrm{NbSe}_{3}$ must be nonplanar. From the resistivity change below $140 \mathrm{~K}$, we estimate that half of the Fermi surface vanishes at the transition.

(iv) In $\mathrm{TaS}_{3}$ the coexistence of the diffuse lines at $0.25 c^{*}$ with satellites was observed, while in $\mathrm{NbSe}_{3}$ the diffuse lines are absent below the transition as shown in Fig. 2. The diffuse streak connecting the parent spots perpendicular to the chain axis was often observed in both materials. It was not determined whether the diffuse streak is due to the soft phonon mode or the poor crystallization.

(v) In $\mathrm{TaS}_{3}$ the satellite spots also have components perpendicular to the chain axis,${ }^{10}$ while in $\mathrm{NbSe}_{3}$ only the component along the chain axis was observed.

We could not extend the electron diffraction study below $130 \mathrm{~K}$ because of the limitation of our cooling apparatus. It must be very interesting to determine the nature of the 59-K anomaly.

We thank Dr. S. Kagoshima of Electrotechnical

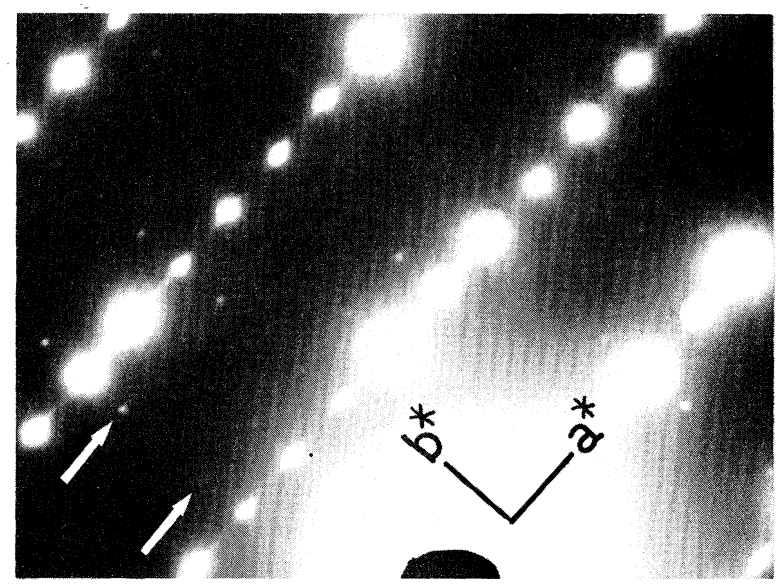

FIG. 2. Electron diffraction pattern of $\mathrm{NbSe}_{3}$ below $140 \mathrm{~K}$, showing the satellite spots (marked by arrows).

Laboratory for valuable discussions and $\mathrm{Mr}$. $\mathrm{H}$. Kuwahata of Department of Metallurgy for his skillful assistance in electron diffraction measurement.

${ }^{1}$ J. Chaussy, P. Haen, J. C. Lasjaunias, P. Monceau, G. Waysand, A. Waintal, A. Meerschaut, P. Molinie, and J. Rouxel, Solid State Commun。20, 759 (1976).

${ }^{2}$ P. Haen, P. Monceau, B. Tissier, G. Waysand, A. Meerschaut, P. Molinie, and J. Rouxel, in Proceedings of the Fourteenth International Conference on Low Temperature Physics, Otaniemi, Finland, 1975, edited by M. Krusius and M. Vuorio (North-Holland, Amsterdam, 1975), Vol. 5, p. 455.

${ }^{3}$ P. Monceau, N. P. Ong, A. M. Portis, A. Meerschaut, and J. Rouxel, Phys. Rev. Lett. 37, 602 (1976).

${ }^{4} \mathrm{~A}$. Meershaut and J。Rouxel, J. Less-Common Met. 39, 197 (1975).

${ }^{5}$ T. Sambongi, M. Yamamoto, K. Tsutsumi, Y. Shiozaki, K. Yamaya, and Y. Abe, J. Phys. Soc. Jpn. 42, 1421 (1977).

${ }^{6} \mathrm{~T}$. Sambongi, K. Tsutsumi, Y. Shiozaki, M. Yamamoto, K. Yamaya, and Y. Abe, Solid State Commun. $\underline{22}$; 729 (1977)。

${ }^{7} \mathrm{E}$. Bjerklund and A. Kjekshus, Z. Anorg. Allg. Chem. 328, 235 (1964).

${ }^{8} \mathrm{~K}$. Bjerklund and A. Kjekshus, Acta Chem. Scand. 19, 701 (1965).

${ }^{9}$ E. Bjerkelund, J. H. Fermor, and A. Kjekshus, Acta Chem. Scand。20, 1836 (1966).

${ }^{10} \mathrm{~K}$. Tsutsumi, T. Sambongi, S. Kagoshima, and $\mathrm{T}$. Ishiguro, to be published. 


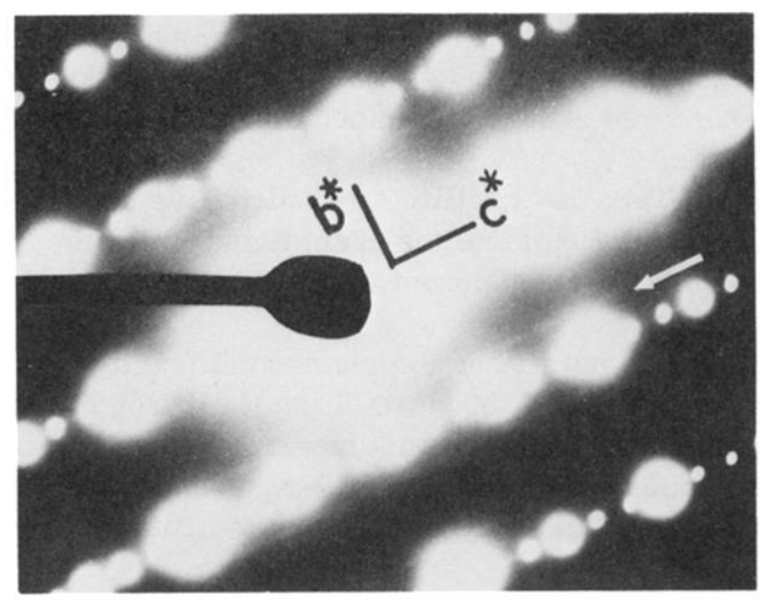

FIG. 1. Electron diffraction pattern of $\mathrm{NbSe}_{3}$ above $140 \mathrm{~K}$, showing one-dimensional anomaly in diffuse scattering (marked by arrows). 


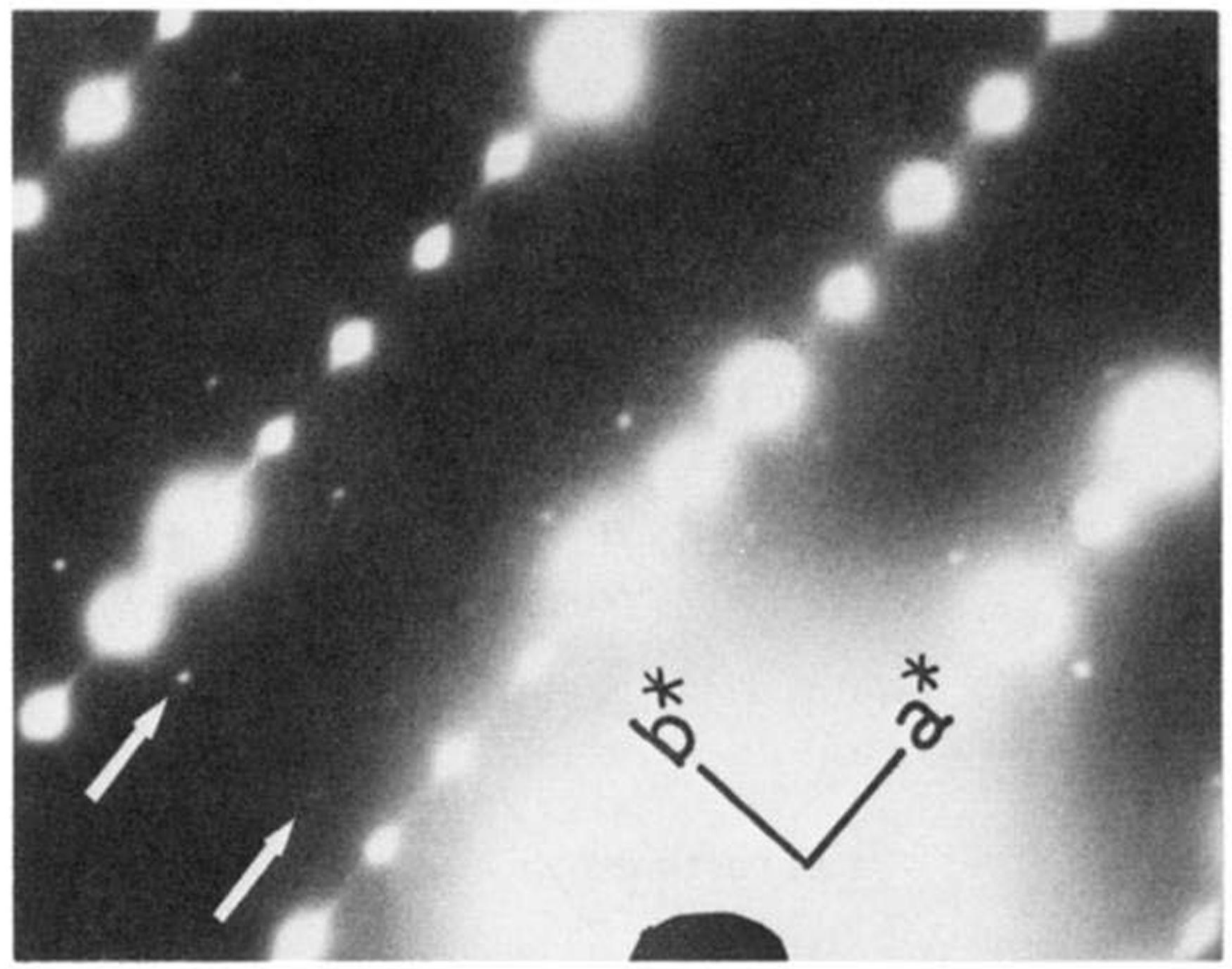

FIG. 2. Electron diffraction pattern of $\mathrm{NbSe}_{3}$ below $140 \mathrm{~K}$, showing the satellite spots (marked by arrows). 